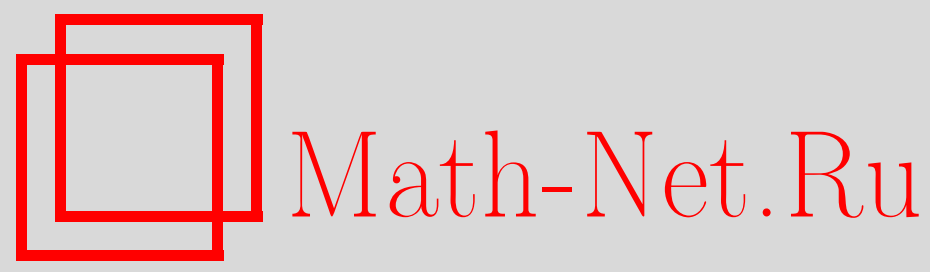

Н. Н. Ганиходжаев, Ф. М. Мухамедов, О некоторых свойствах одного класса диагонализуемых состояний неймановских алгебр, Матем. заметки, 2004, том 76, выпуск 3, 350361

DOI: https://doi.org/10.4213/mzm111

Использование Общероссийского математического портала Math-Net.Ru подразумевает, что вы прочитали и согласны с пользовательским соглашением http://www.mathnet.ru/rus/agreement

Параметры загрузки:

IP : 35.174 .16 .151

26 апреля 2023 г., $17: 03: 35$

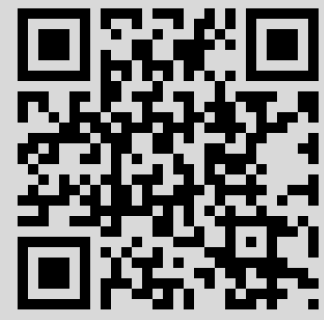




\title{
О НЕКОТОРЫХ СВОЙСТВАХ ОДНОГО КЛАССА ДИАГОНАЛИЗУЕМЫХ СОСТОЯНИЙ НЕЙМАНОВСКИХ АЛГЕБР
}

\author{
Н. Н. Ганиходжаев, Ф. М. Мухамедов
}

В данной работе строится один класс представлений равномерно гиперфинитных алгебр и изучаются соответствующие им алгебры фон Неймана. Доказано, что марковские состояния при некоторых условиях порождают в представлении ГНС факторы типа $\mathrm{III}_{\lambda}$, где $\lambda \in(0,1)$, что дает отрицательный ответ гипотезе о том, что факторы соответствующие гамильтонианам с нетривиальными взаимодействиями, имеют тип $\mathrm{III}_{1}$. Показано, что для одного класса гамильтонианов существует единственное трансляцонно-инвариантное основное состояние.

Библиографой: 16 названий.

1. Введение. В данной работе строится один класс представлений равномерно гиперфинитных алгебр и изучаются соответствующие им алгебры фон Неймана. Серию представлений, построенных Пауэрсом [1] по product-мерам, можно трактовать как свободную решетчатую квантовую спиновую систему с двумя состояниями в каждой точке, причем первое состояние занято с вероятностью $p$, а второе - с вероятностью $q=1-p$; тогда соответствующая алгебра фон Неймана является фактором типа $\mathrm{III}_{\lambda}$, где $\lambda=p / q$.

В [2] высказьвается предположение, что “по-видимому (в настоящее время не доказано), пример Пауэрса [1] является нетипичным для квантовых спиновых систем (с нетривиальньми взаимодействиями) и всегда следует ожидать появления факторов типа $\mathrm{III}_{1} \ldots "$.

Во втором пункте приводятся необходимые сведения из теории алгебр фон Неймана. В третьем пункте данной работы приводится пример, опровергаюший вышеупомянутую гипотезу. В четвертом пункте доказывается единственность трансляционно-инвариантного основного состояния для одного класса гамильтонианов.

2. Предварительные сведения и определения. В этом пункте приведем некоторые необходимые факты из теории алгебр фон Неймана. Пусть $B(H)$ - алгебра всех ограниченных линейных операторов на гильбертовом пространстве $H$ (над полем комплексных чисел).

Замкнутая в слабой операторной топологии $*$-подалгебра $M$ в $B(H)$ называется $a \Omega$ геброй фон Неймана, если она содержит тождественный оператор 1. Через $\operatorname{Proj}(M)$ обозначается множество всех проекторов на $M$. Алгебру фон Неймана называют фактором, если ее центр $Z(M)=\{x \in M: x y=y x \quad \forall y \in M\}$ тривиален, т.е.

$$
Z(M)=\{\lambda \mathbf{1} \mid \lambda \in C\} \text {. }
$$


Алгебры фон Неймана классифицируются по типам I $\left(\mathrm{I}_{n}, n<\infty, \mathrm{I}_{\infty}\right), \mathrm{II}\left(\mathrm{II}_{1}, \mathrm{II}_{\infty}\right)$ и III (подробности можно найти, например, в [3], [4]).

Пусть $M$ - алгебра фон Неймана. Линейное биективное отображение $\alpha: M \rightarrow M$ назьвается *-автоморфизмом, если $\alpha\left(x^{*}\right)=\alpha(x)^{*}$ и $\alpha(x y)=\alpha(x) \alpha(y)$ для всех $x, y \in M$. Линейный функционал $\omega$ на $M$ назьвается положительным, если $\omega\left(x^{*} x\right) \geqslant 0$ для всех $x \in M$. Положительный функционал $\omega$ на $M$ с $\|\omega\|=1$ назьвается состоянием. Состояние $\omega$ назьвается следом, если $\omega(x y)=\omega(y x)$ для всех $x, y \in M$.

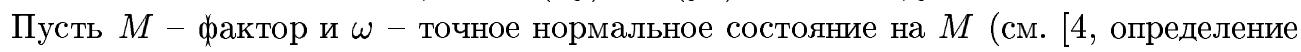
2.4.20]) и $\sigma_{t}^{\omega}$ - модулярная группа, ассоциированная с $\omega$ на $M$ (см. [4, определение 2.5.15]). Через $\Gamma\left(\sigma^{\omega}\right)$ обозначим спектр Конна модулярной групшы $\sigma_{t}^{\omega}$ (см. [4], [5, определение 2.2.1]).

ОПРЕДЕЛЕНИЕ 2.1 [5]. Фактор $M$ имеет тип:

(i) $\mathrm{III}_{1}$, если $\Gamma\left(\sigma^{\omega}\right)=\mathbb{R}$;

(ii) $\mathrm{III}_{\lambda}$, если $\Gamma\left(\sigma^{\omega}\right)=\{n \ln \lambda, n \in \mathbb{Z}\}, \lambda \in(0,1)$;

(iii) $\mathrm{III}_{0}$, если $\Gamma\left(\sigma^{\omega}\right)=\{0\}$.

Рассмотрим $C^{*}$-алгебру $A=\bigotimes_{\mathbb{Z}} M_{2}(\mathbb{C})$, где $M_{2}(\mathbb{C})$ - алгебра матриц $2 \times 2$ над полем комплексных чисел $\mathbb{C}$. Взаимодействием назьвается функция $\Phi$, сопоставляющая конечным подмножествам $X \subset \mathbb{Z}$ самосопряженные элементы $A$ такие, что $\Phi(X) \in A_{X}$ $\left(=\bigotimes_{x \in X} M_{2}(\mathbb{C})\right)$. Заметим, что конечным подмножествам $X \subset \mathbb{Z}$ отвечают полные матричные алгебры $\bigotimes_{x \in X} A_{x}, A_{x}=M_{2}(\mathbb{C})$, которые изоморфны подалгебрам $A_{X} \subset A$, а потому и (формально) отождествляются с ними. Каждое $\Phi(X)$ представляет энергию взаимодействия множества всех частиц в конечном подмножестве $X$. Общая энергия $H_{\Phi}(\Lambda)$ в объеме $\Lambda \subset \mathbb{Z}$ определяется как

$$
H_{\Phi}(\Lambda)=\sum_{X \subseteq \Lambda} \Phi(X),
$$

и она называется гамильтонианом, ассоциированным $c \Lambda$. Через $H_{\Phi}$ обозначается энергия системы, т.е. $H_{\Phi}=H_{\Phi}(\mathbb{Z})$. В этом случае $H_{\Phi}$ рассматривается как формальный ряд. Положим

$$
A_{\mathrm{loc}}=\bigcup_{n \in \mathbb{N}} \bigotimes_{j=-n}^{n} M_{2}(\mathbb{C}) .
$$

Предположим, что для гамильтониана $H_{\Phi}$ можно определить однопараметрическую группу автоморфизмов на $A$ следующим образом:

$$
\gamma_{t}(x)=\lim _{\Lambda \rightarrow \mathbb{Z}} e^{i t H_{\Phi}(\Lambda)} x e^{-i t H_{\Phi}(\Lambda)}
$$

где $t \in \mathbb{R}$ и $x$ из $A$. Состояние $\omega$ на $A$ назьвается основным состоянием относительно $H_{\Phi}$, если выполнено условие

$$
\left.\frac{1}{i} \frac{d}{d t} \omega\left(x^{*} \gamma_{t}(x)\right)\right|_{t=0} \geqslant 0
$$

для всех $x$ из $A_{\text {loc }}$. 
Напомним, что некоммутативное марковское состояние впервые было введено в [6] и определяется следующим образом (см. [6]): пусть $N$ - алгебра матриц $n \times n, \varphi_{1}(\cdot)=$ $\operatorname{tr}(\rho(\cdot))$ - состояние на $N$, где $\operatorname{tr}$ - след на $N$ и $\rho$ - положительньй оператор с единичным следом. Пусть $T: N \rightarrow N$ - линейное отображение такое, что $\varphi_{1}(x)=\varphi_{1}(T x)$ для всех $x \in N$. В алгебре

$$
\bigotimes^{n} N=\underbrace{N \otimes N \otimes \cdots \otimes N}_{n}
$$

определяется линейньй функционал

$$
\varphi_{n}\left(a_{1} \otimes a_{2} \otimes \cdots \otimes a_{n}\right)=\varphi_{1}\left(a_{1} T\left(a_{2}\left(\ldots T\left(a_{n}\right) \ldots\right)\right)\right)
$$

Если отображение $T$ таково, что $\varphi_{n}-$ состояние, то говорят, что $\varphi_{n}$ является марковским состоянием. В алгебре $\bigotimes^{\infty} N=\bigcup_{n} \otimes^{n} N$ марковское состояние определяется как $\varphi_{\infty}=\bigcup_{k=1}^{\infty} \varphi_{k}$. Заметим, что более общее определение марковского состояния было введено в [7].

Пусть $P=\left(p_{i j}\right)_{i, j=1}^{2}-$ стохастическая матрица, т.е. $p_{i j} \geqslant 0$ и $\sum_{j=1}^{2} p_{i j}=1, i=1,2$, такая, что $p_{i j}>0$ для всех $i$ и $j$. Пусть далее $\pi=\left(p_{1}, p_{2}\right)-$ вектор такой, что $p_{1}+p_{2}=1$, $p_{1} \geqslant 0, p_{2} \geqslant 0$, и $\pi P=\pi$, а $e_{1}\left(=e_{11}\right), e_{2}\left(=e_{22}\right), e_{3}\left(=e_{12}\right), e_{4}\left(=e_{21}\right)$ - матричные единищы алгебры $M_{2}(\mathbb{C})$. Через $\mathbb{C} M_{2}(\mathbb{C})$ обозначим коммутативную подалгебру $M_{2}(\mathbb{C})$, порожденную элементами $e_{1}$ и $e_{2}$.

Рассмотрим $C^{*}$-алгебру $A=\bigotimes_{\mathbb{Z}} M_{2}(\mathbb{C})$ и положим $\mathbb{C} A=\bigotimes_{\mathbb{Z}} \mathbb{C} M_{2}(\mathbb{C})$. Элементы коммутативной алгебры $\mathbb{C} A$ будем представлять как функции на пространстве $\Omega=$ $\left\{e_{1}, e_{2}\right\}^{\mathbb{Z}}$. Пусть на измеримом пространстве $(\Omega, \Sigma)$ задана мера $\mu$, где $\Sigma-\sigma$-алгебра, порожденная конечномерными цилиндрическими подмножествами $\Omega$. Построим состояние $\omega_{\mu}$ на $A$ следующим образом. Положим $\omega_{\mu}(x)=0$, если тензорный одночлен $x$ от матричных единиц $e_{i}, i=1, \ldots, 4$, содержит, по крайней мере, одну частичную изометрию. Если же $x \in \mathbb{C} A$, то положим

$$
\omega_{\mu}(x)=\int_{\Omega} x d \mu
$$

Состояние, построенное таким образом, было введено в [8], и называлось диагональнblм.

Рассмотрим марковскую меру $\mu_{P}$ на $(\Omega, \Sigma)$, определенную с помощью стохастической матрищы $P$ следующим образом:

$$
\mu_{P}\left\{x=\left(x_{n}\right) \in \Omega \mid x_{k+1}=e_{i_{1}}, x_{k+2}=e_{i_{2}}, \ldots, x_{k+n}=e_{i_{n}}\right\}=p_{i_{1}} p_{i_{1} i_{2}} \cdots p_{i_{n-1} i_{n}}
$$

где $i_{k} \in\{1,2\}, k \in \mathbb{Z}$. Через $\omega_{P}$ обозначим диагональное состояние, порожденное марковской мерой $\mu_{P}$. Отметим, что состояние $\omega_{P}$ является марковским состоянием на $A$. Действительно, в нашем случае $N=M_{2}(\mathbb{C}), \rho=\left(\begin{array}{cc}p_{1} & 0 \\ 0 & p_{2}\end{array}\right)$, a $T: M_{2}(\mathbb{C}) \rightarrow M_{2}(\mathbb{C})$ определяется следующим образом:

$$
T\left(\begin{array}{ll}
a & b \\
c & d
\end{array}\right)=\left(\begin{array}{cc}
p_{11} a+p_{12} d & 0 \\
0 & p_{21} a+p_{22} d
\end{array}\right)
$$


Через $e_{i}^{(n)}, n \in \mathbb{Z}, i \in\{1,2,3,4\}$, обозначим матричные единицы алгебры $M_{2}(\mathbb{C})$, находящиеся на $n$-м месте в произведении $\bigotimes_{\mathbb{Z}} M_{2}(\mathbb{C})$. Тогда $*$-автоморфизм $\beta: A \rightarrow A$ называется сдвигом, если для любых $n \in \mathbb{Z}$ и $i \in\{1,2,3,4\}$ имеет место $\beta\left(e_{i}^{(n)}\right)=e_{i}^{(n+1)}$. Состояние $\omega$ на $A$ называется трансляционно-инвариантным, если выполнено равенство

$$
\omega(\beta(x))=\omega(x) \quad \text { для всех } x \in A .
$$

Заметим, что марковское состояние $\omega_{P}$ является транслящионно-инвариантным.

3. О типе алгебры фон Неймана, порождаемой марковским состоянием при ассоциированном представлении. Рассмотрим на $C^{*}$-алгебре $A=\bigotimes_{\mathbb{Z}} M_{2}(\mathbb{C})$ марковское состояние $\omega_{P}$, определенное с помощью стохастической матрицы $P=$ $\left(p_{i j}\right)_{i, j=1}^{2}$. На конечномерной $C^{*}$-подалгебре $A_{[-n ; n]} \subset A$ перепишем состояние $\omega_{P}$ в виде

$$
\omega_{P}(x)=\frac{\operatorname{tr}\left(e^{H([-n ; n], \partial)} x\right)}{\operatorname{tr}\left(e^{H([-n ; n], \partial)}\right)}, \quad x \in A_{[-n ; n]},
$$

где $\operatorname{tr}$ - след на $A_{[-n ; n]}$. Из $(1)$, пользуясь результатом $[9$, гл. $1, \S 2$, п. 1], вид гамильтониана $H([-n ; n], \partial)$ в стандартном базисе в $A_{[-n, n]}$ (т.е. относительно матричных единиц) представляется как

$$
\begin{aligned}
H([-n ; n], \partial) & =-\sum_{i=-n}^{n-1} \Phi_{i, i+1}+\left(\begin{array}{cc}
\ln p_{1} & 0 \\
0 & \ln p_{2}
\end{array}\right) \underset{i=-n+1}{\bigotimes} \mathbf{1}, \\
\Phi_{i, i+1} & =\left(\begin{array}{cccc}
\ln p_{11} & 0 & 0 & 0 \\
0 & \ln p_{12} & 0 & 0 \\
0 & 0 & \ln p_{21} & 0 \\
0 & 0 & 0 & \ln p_{22}
\end{array}\right)
\end{aligned}
$$

где $\Phi_{i, i+1} \in A_{[i, i+1]}$, т.е. оно записьвается следующим образом:

$$
\Phi_{i, i+1}=\sum_{m, l=1}^{2} \ln p_{m l} e_{m}^{(i)} \otimes e_{l}^{(i+1)} .
$$

Известно (см. [10], [11]), что на $\mathbb{Z}$ для потенциалов конечного радиуса взаимодействия существует единственное предельное гиббсовское состояние. В силу этого потенциалу

$$
H=-\sum_{i \in \mathbb{Z}} \Phi_{i, i+1}
$$

который далее будем называть марковским гамильтонианом, отвечает единственное предельное гиббсовское состояние $\omega$ на $A$. В силу (2) и единственности предельного состояния $\omega$ следует, что $\omega=\omega_{P}$. Следовательно, $\omega_{P}$ является фактор-состоянием (см. [10, теорема 5.3.30]). Положим $M=\pi_{\omega_{P}}(A)^{\prime \prime}$, где $\pi_{\omega_{P}}$ - представление, ассоциированное с состоянием $\omega_{P}$ (см. [4, определение 2.3.18]). Определим теперь тип фактора $M$. Модулярная групша на $M$ определяется следующим образом:

$$
\sigma_{t}^{\omega_{P}}(x)=\lim _{\Lambda \rightarrow \mathbb{Z}} e^{i t H(\Lambda)} x e^{-i t H(\Lambda)} .
$$


Сушествование этого предела доказано в [10, теорема 6.2.4]. Через $M_{\omega_{P}}$ обозначим централизатор состояния $\omega_{P}$, который определяется как

$$
M_{\omega_{P}}=\left\{x \in M: \omega_{P}(x y)=\omega_{P}(y x) \text { для всех } y \in M\right\} .
$$

Так как гиббсовское состояние $\omega_{P}$ является точным нормальным состоянием на $M$ и гамильтониан определяет модулярную групу, отсюда следует совпадение централизатора $M_{\omega_{P}}$ точного нормального состояния $\omega_{P}$ с подалгеброй неподвижных точек соответствующей модулярной группы (см. [10, предложение 5.3.28]):

$$
M^{\sigma}=\left\{x \in M: \sigma_{t}^{\omega_{P}}(x)=x \quad \forall t \in \mathbb{R}\right\} .
$$

Через $\Pi[n]$ обозначим группу перестановок множества $[-n, n] \cap \mathbb{Z}$, состоящую из элементов $\gamma$ таких, что

$$
\gamma(k)=k \quad \forall k \in\{-n, n\} .
$$

Каждый элемент $\gamma \in \Pi[n]$ определяет автоморфизм $\alpha_{\gamma}: M \rightarrow M$, который определяется следуюшим образом:

$$
\alpha_{\gamma}\left(\prod_{k \in[-n, n]}^{\otimes} a_{k}\right)=\prod_{k \in[-n, n]}^{\otimes} a_{\gamma(k)},\left.\quad \alpha_{\gamma}\right|_{\bigotimes_{k \notin[-n, n]} M_{q}(\mathbb{C})}=\mathrm{id} .
$$

Обозначим

$$
\mathscr{S}_{0}=\bigcup_{n \in \mathbb{N}}\left\{\alpha_{\gamma} \mid \gamma \in \Pi[n]\right\} .
$$

В [12] доказано следующее

ПРЕДЛОЖЕНИЕ 3.1. Групna

$$
G_{0}=\left\{\alpha \in \mathscr{S}_{0} \mid \omega_{P}(\alpha(x))=\omega_{P}(x) \forall x \in M\right\}
$$

действует әргодическим образом на $M$, т.е. из равенства $\alpha(x)=x \forall \alpha \in G_{0}$ cледует $x=\theta \mathbf{1}, \theta \in \mathbb{C}$.

Справедлива следующая

Лемма 3.1. Пусть $P=\left(p_{i j}\right)_{i, j=1}^{2}$ - стохастическая матрица такая, что $p_{i j}>0$, $i, j=1,2$, и хотя бы один әлемент әтой матрицы отличен от 1/2, и пусть $\omega_{P}$ - соответствующее матрице $P$ марковское состояние на $A$. Тогда $M^{\sigma}$ есть фактор muna $\mathrm{II}_{1}$.

ДокАЗАТЕЛьСтво. Из определения автоморфизма $\alpha_{\gamma}$ (см. (6)) следует, что каждый автоморфизм $\alpha \in G_{0}$ является внутренним, т.е. существует унитарный оператор $u_{\alpha} \in M$ такой, что $\alpha(x)=u_{\alpha} x u_{\alpha}^{*}, x \in M$. Из условия $\omega_{P} \circ \alpha=\omega_{P}$ находим

$$
\omega_{P}\left(u_{\alpha} x u_{\alpha}^{*}\right)=\omega_{P}(x) \quad \forall x \in M .
$$

Из (5) следует, что $u_{\alpha} \in M_{\omega_{P}}$. В силу предложения 3.1 групша $G_{0}$ действует эргодическим образом, т.е. из справедливости равенства $u_{\alpha} x=x u_{\alpha}$ для всех $\alpha \in G_{0}$ следует, что $x=\theta \mathbf{1}, \theta \in \mathbb{C}$. Следовательно, $\left\{u_{\alpha} \mid \alpha \in G_{0}\right\}^{\prime}=\mathbb{C} \mathbf{1}$. Из включения $M^{\sigma \prime} \subset\left\{u_{\alpha}\right\}^{\prime}$ получим

$$
M^{\sigma \prime} \cap M=\mathbb{C} \mathbf{1} \text {. }
$$

В частности, $M^{\sigma \prime} \cap M^{\sigma}=\mathbb{C} 1$. Это означает, что $M^{\sigma}$ есть фактор. Из того, что сужение состояния $\omega_{P}$ на $M_{\omega_{P}}$ есть след и из включения $\mathbb{C} A \subset M^{\sigma}$ находим, что $M^{\sigma}$ имеет тип $\mathrm{II}_{1}$. Лемма доказана. 
ЗАмЕчАниЕ 1. Аналогичная лемма для более обшего класса марковских состояний была доказана в [13].

Известно (см. [5, предложение 2.2.2]), что спектр Конна $\Gamma(\alpha)$ группы автоморфизмов $\alpha=\left\{\alpha_{g}\right\}_{g \in G}$ алгебры фон Неймана $M$ имеет следующий вид:

$$
\Gamma(\alpha)=\cap\left\{\operatorname{Sp}\left(\alpha^{e}\right) \mid e \in \operatorname{Proj}\left(Z\left(M^{\alpha}\right)\right), e \neq 0\right\},
$$

где $Z\left(M^{\alpha}\right)$ - центр подалгебры

$$
M^{\alpha}=\left\{x \in M: \alpha_{g}(x)=x \forall g \in G\right\},
$$

$\alpha^{e}(x)=\alpha($ exe $), x \in e M e$. Здесь $\operatorname{Sp}(\alpha)-$ спектр Арвесона группы автоморфизмов $\alpha$ (см. более подробно [5], [14]). Из леммы 3.1 следует, что $Z\left(M^{\sigma}\right)=\mathbb{C} 1$. Отсюда в силу равенства $(7)$ имеем $\Gamma\left(\sigma^{\omega_{P}}\right)=\operatorname{Sp}\left(\sigma^{\omega_{P}}\right)$.

Пусть $\Lambda=[-n, n]$ и рассмотрим

$$
H(\Lambda)=\sum_{i=-n}^{n} \Phi_{i, i+1} .
$$

Через $\operatorname{Sp}(H(\Lambda))$ обозначим спектр $H(\Lambda)$. Если положить

$$
\sigma_{t}^{\omega_{P}, \Lambda}(x)=e^{i t H(\Lambda)} x e^{-i t H(\Lambda)},
$$

TO

$$
\operatorname{Sp}\left(\sigma^{\omega_{P}, \Lambda}\right)=\operatorname{Sp}(H(\Lambda))-\operatorname{Sp}(H(\Lambda))=\{\lambda-\mu: \lambda, \mu \in \operatorname{Sp}(H(\Lambda))\} .
$$

Положим $\lambda_{1}=\ln p_{11}, \lambda_{2}=\ln p_{12}, \lambda_{3}=\ln p_{21}, \lambda_{4}=\ln p_{22}$. Поскольку $H(\Lambda)$ имеет следуюший вид:

$$
H(\Lambda)=\sum_{i=-n}^{n-1} \bigotimes_{j \notin\{i, i+1\}} \mathbf{1} \otimes \Phi_{i, i+1},
$$

из (3) следует, что $\operatorname{Sp}\left(\sigma^{\omega_{P}, \Lambda}\right)$ порождается элементами вида

$$
\lambda_{1}-\lambda_{2}, \lambda_{1}-\lambda_{3}, \lambda_{1}-\lambda_{4}, \lambda_{2}-\lambda_{3}, \lambda_{2}-\lambda_{4}, \lambda_{3}-\lambda_{4} .
$$

Taк как

$$
\frac{p_{11}}{p_{22}}=\frac{p_{11}}{p_{12}} \frac{p_{12}}{p_{22}}, \quad \frac{p_{12}}{p_{21}}=\frac{p_{12}}{p_{22}} \frac{p_{22}}{p_{21}}
$$

то $\operatorname{Sp}(H(\Lambda))$ порождается

$$
\ln \frac{p_{11}}{p_{12}}, \ln \frac{p_{11}}{p_{21}}, \ln \frac{p_{12}}{p_{22}}, \ln \frac{p_{21}}{p_{22}} .
$$

Если существует такое наименьшее $\lambda \in(0,1)$, что

$$
\frac{p_{11}}{p_{12}}=\lambda^{m_{1}}, \quad \frac{p_{11}}{p_{21}}=\lambda^{m_{2}}, \quad \frac{p_{12}}{p_{22}}=\lambda^{m_{3}}, \quad \frac{p_{21}}{p_{22}}=\lambda^{m_{4}},
$$

где $m_{j} \in \mathbb{Z}, j=1, \ldots, 4$, то $\operatorname{Sp}(H(\Lambda))=\{n \ln \lambda\}_{n=-m}^{m}$, откуда следует, что

$$
\operatorname{Sp}\left(\sigma^{\omega_{P}}\right)=\{n \ln \lambda\}_{n \in \mathbb{Z}} .
$$

Таким образом, в этом случае $\pi_{\omega_{P}}(A)^{\prime \prime}$ имеет тип $\mathrm{III}_{\lambda}$ и, если условие $(8)$ не имеет места, то из $\Gamma\left(\sigma^{\omega_{P}}\right) \neq 0$ следует, что $\Gamma\left(\sigma^{\omega_{P}}\right)=\mathbb{R}$. Итак, доказана следующая 
ТЕОремА 3.1. Пусть $P=\left(p_{i j}\right)_{i, j=1}^{2}-$ стохастическая матрииа такая, что $p_{i j}>0, i, j=1,2$, и хотя бы один әлемент әтой матрицы отличен от $1 / 2$, и пусть $\omega_{P}$ - соответствующее матрице $P$ марковское состояние. Если существует наименьшее $\lambda \in(0,1)$ такое, что справедливо $(8)$, то $\pi_{\omega_{P}}(A)^{\prime \prime}$ - фактор типа $\mathrm{III}_{\lambda}$, а в противном случае - фактор типа $\mathrm{III}_{1}$.

ЗАмЕчаниЕ 2. Если все элементы стохастической матрицы $P$ равны $1 / 2$, то для соответствующего марковского состояния $\omega_{P}$ фактор $\pi_{\omega_{P}}(A)^{\prime \prime}$ имеет тип $\mathrm{II}_{1}$ (см. [15]).

ЗАмечАниЕ 3. Рассматриваемые здесь марковские состояния отличны от состояний, изученных в [16]. Действительно, пусть $\omega_{R}-$ состояние на алгебре канонических антикоммутационных соотношений (KAC), построенное по положительному сжатию $\mathscr{R}=q \mathbf{1}+A$, где $0<q<1 / 2$ и $A$ - оператор Гильберта-Шмидта. Тогда состояние $\omega_{\mathscr{R}}$ порождает модулярную группу $\sigma^{\omega_{\mathscr{R}}}$ со спектром Арвесона, которьй может быть достаточно сложным в зависимости от $A$, и, в то же время, спектр Конна имеет вид $\left\{(q /(1-q))^{n}, n \in \mathbb{Z}\right\}$. Таким образом, это состояние определяет фактор типа $\mathrm{III}_{\lambda}$, где $\lambda=q /(1-q)$.

Пусть $l_{1}, l_{2} \in \mathbb{R}$. Положим

$$
\left(l_{1}, l_{2}\right)=\max \left\{|l|: \frac{l_{1}}{l} \in \mathbb{Z}, \frac{l_{2}}{l} \in \mathbb{Z}\right\}
$$

Обозначим

$$
\frac{p_{11}}{p_{12}}=e^{-l_{1}}, \quad \frac{p_{11}}{p_{21}}=e^{-l_{2}}, \quad \frac{p_{12}}{p_{22}}=e^{-l_{3}}, \quad \frac{p_{21}}{p_{22}}=e^{-l_{4}}
$$

ТЕОРемА 3.2. Пусть выполнены все условия теоремы 3.1. Eсли $l_{i} / l_{i+1} \in \mathbb{Q}$, $i=1,2,3$, то $\pi_{\omega_{P}}(A)^{\prime \prime}$ uмеет mun $\mathrm{III}_{\lambda}$, где

$$
\lambda=e^{-\left(\left(l_{1}, l_{2}\right),\left(l_{3}, l_{4}\right)\right)} .
$$

В противном случае $\pi_{\omega_{P}}(A)^{\prime \prime}$ имеет тип $\mathrm{III}_{1}$.

Приведем примеры, которые удовлетворяют и не удовлетворяют условию (8).

ПРИмеР 3.1. Пусть $p \in(0,1)$. Рассмотрим стохастическую матрицу

$$
P=\frac{1}{1+p}\left(\begin{array}{ll}
1 & p \\
1 & p
\end{array}\right)
$$

Легко видеть, что марковское состояние $\omega_{p}$, соответствующее стохастической матрице $P$, является состоянием Пауэрса (см. [1]). Нетрудно проверить, что справедливо равенство (8) с параметрами $m_{1}=0, m_{2}=-1, m_{3}=1, m_{4}=0$ и $\lambda=p$. Тогда из теоремы 3.1 следует, что состояние $\omega_{P}$ порождает фактор типа $\mathrm{III}_{\lambda}$, т.е. теорема 3.1 согласуется с теоремой Пауэрса (см. [1, теорема 4.8]). 
ПримеР 3.2. Рассмотрим уравнение $x^{5}-x^{3}-x+1=0$ или $(x-1)\left(x^{3}(x+1)-1\right)=0$. Пусть $\mu$ есть решение уравнения $x^{3}(x+1)-1=0$, принадлежащее интервалу $(0,1)$. Определим стохастическую матрицу

$$
p_{11}=\frac{1}{1+\mu^{5}}, \quad p_{21}=\frac{1}{1+\mu^{2}}, \quad p_{12}=1-p_{11}, \quad p_{22}=1-p_{21}
$$

Тогда нетрудно убедиться в справедливости равенства (8) с параметрами $\lambda=\mu, m_{1}=$ $-5, m_{2}=-1, m_{3}=-2, m_{4}=2$. Из теоремы 3.1 следует, что марковское состояние, порожденное матрицей $P$, порождает фактор типа $\mathrm{III}_{\mu}$.

ПримеР 3.3. Определим стохастическую матрицу следующим образом: $p_{11}=1 / 3$, $p_{12}=2 / 3, p_{21}=1 / 4, p_{22}=3 / 4$; тогда легко проверить, что условие $(8)$ не имеет места и, следовательно, по теореме $3.1 \pi_{\omega_{P}}(A)^{\prime \prime}$ имеет тип $\mathrm{III}_{1}$.

Пусть $\Sigma$ - совокупность всех стохастических матриц. На этом множестве определим отображение $\gamma: \Sigma \rightarrow[0,1] \times[0,1]$, полагая

$$
\gamma\left(\begin{array}{ll}
p_{11} & 1-p_{11} \\
p_{21} & 1-p_{21}
\end{array}\right)=\left(p_{11}, p_{21}\right)
$$

Из определения отображения $\gamma$ следует, что оно является взаимнооднозначньм отображением. Пусть $F$ - борелевская $\sigma$-алгебра на $[0,1] \times[0,1]$ и $\nu$ - лебеговская мера на $([0,1] \times[0,1], F)$. На измеримом пространстве $\left(\Sigma, \gamma^{-1}(F)\right)$ определим лебеговскую меpy

$$
\nu_{\Sigma}(B)=\nu(\gamma(B))
$$

где $B \in \gamma^{-1}(F)$.

Множество всех стохастических матрищ, удовлетворяющих условию (8), обозначим через $\Sigma_{0}$, а через $\Delta_{m}$, где $m=\left(m_{1}, m_{2}, m_{3}, m_{4}\right) \in \mathbb{Z}^{4}$, обозначим совокупность всех стохастических матрищ, удовлетворяющих условию (8) при фиксированных значениях $m_{j}$, где $j=1, \ldots, 4$. Отсюда следует

$$
\Sigma_{0}=\bigcup_{m \in \mathbb{Z}^{4}} \Delta_{m}
$$

Так как $\gamma\left(\Delta_{m}\right)$ содержится на некоторой “кривой” на $[0,1] \times[0,1]$, то $\nu\left(\gamma\left(\Delta_{m}\right)\right)=0$. В силу $\sigma$-аддитивности $\nu_{\Sigma}$ находим $\nu_{\Sigma}\left(\Sigma_{0}\right)=0$. Итак, доказана следующая

ТеОрема 3.3. Множество $\Sigma_{0}$ всех стохастических матрии, удовлетворяющих условию (8), имеет лебегову меру нуль, т.е.

$$
\nu_{\Sigma}\left(\Sigma_{0}\right)=0
$$




\section{4. О единственности трансляционно-инвариантного основного состоя-} ния для марковского гамильтониана. В этом пункте дается описание трансляцонно-инвариантных основных состояний для марковского гамильтониана $H$. Пусть на $C^{*}$-алгебре $A=\bigotimes_{\mathbb{Z}} M_{2}(\mathbb{C})$ задано состояние $\omega$. Напомним, что состояние $\omega$ назьвается трансляиионно-инвариантным (см. п. 2), если вьполнено равенство

$$
\omega(\beta(x))=\omega(x) \quad \text { для всех } \quad x \in A .
$$

Из [10, теорема 6.2.58] следует, что трансляционно-инвариантное состояние $\omega$ является основным состоянием тогда и только тогда, когда оно минимизирует следующий функционал:

$$
H(\omega)=\sum_{X \ni 0} \frac{\omega(\Phi(X))}{|X|} .
$$

Учитывая (4) и трансляционную инвариантность состояния $\omega$, вычислим $H(\omega)$ :

$$
H(\omega)=-\frac{\omega\left(\Phi_{-1,0}\right)}{2}-\frac{\omega\left(\Phi_{0,1}\right)}{2}=\omega\left(\Phi_{0,1}\right)=-\sum_{m, l=1}^{2} \ln p_{m l} \omega\left(e_{m} \otimes e_{l}\right) .
$$

Положим $a_{i j}=\omega\left(e_{i} \otimes e_{j}\right), i, j=1,2$. Из равенств

$$
\omega\left(e_{1} \otimes e_{2}\right)+\omega\left(e_{1} \otimes e_{1}\right)=\omega\left(e_{1}\right)=\omega\left(e_{2} \otimes e_{1}\right)+\omega\left(e_{1} \otimes e_{1}\right)
$$

следует

$$
a_{i j}=a_{j i} \quad \text { при } i \neq j .
$$

В силу сказанного вьше мы приходим к следующей вариационной задаче:

$$
\left[\begin{array}{c}
-\sum_{i, j=1}^{2} a_{i j} \ln p_{i j} \rightarrow \min \\
\sum_{i, j=1}^{2} a_{i j}=1, \quad a_{i j} \geqslant 0 .
\end{array}\right.
$$

Имея в виду (9), получим

$$
\sum_{i, j=1}^{2} a_{i j} \ln p_{i j}=\ln \frac{p_{11}}{p_{22}} a_{11}+\ln \frac{p_{12} p_{21}}{p_{22} p_{22}} a_{12}+\ln p_{22} .
$$

Положим

$$
C=\ln \frac{p_{11}}{p_{22}}, \quad D=\ln \frac{p_{12} p_{21}}{p_{22} p_{22}} .
$$

Тогда задача (10) эквивалентна следующей задаче:

$$
\left[\begin{array}{l}
C a_{11}+D a_{12} \rightarrow \max , \\
0 \leqslant 1-a_{11}-2 a_{12} \leqslant 1 \\
a_{11}, a_{12} \geqslant 0 .
\end{array}\right.
$$

Рассмотрим следующие случаи:

(i) $p_{11}>p_{22}, p_{21} \leqslant p_{22}$;

(ii) $p_{11}<p_{22}, p_{22} \leqslant p_{21}$;

(iii) $p_{11} \cdot p_{21} \geqslant p_{22}^{2}$. 
Тогда из (11) соответственно имеем:

(i) $C>0, D<0$;

(ii) $C<0, D>0$;

(iii) $C>0, D>0$.

Рассмотрим случай (i). Положим $D_{1}=-D$. Отсюда (12) имеет следующий вид:

$$
\left[\begin{array}{l}
C a_{11}-D_{1} a_{12} \rightarrow \max \\
0 \leqslant 1-a_{11}-2 a_{12} \leqslant 1 \\
a_{11}, a_{12} \geqslant 0 .
\end{array}\right.
$$

Очевидно, что решение (13) есть $a_{11}=1, a_{12}=0$, т.е.

$$
\omega\left(e_{1} \otimes e_{1}\right)=1, \quad \omega\left(e_{1} \otimes e_{2}\right)=\omega\left(e_{2} \otimes e_{1}\right)=\omega\left(e_{2} \otimes e_{2}\right)=0 .
$$

Аналогично, рассматривая остальные (ii) и (iii) случаи, соответственно получим следуюшие равенства:

$$
\begin{aligned}
& \omega\left(e_{1} \otimes e_{1}\right)=0, \quad \omega\left(e_{1} \otimes e_{2}\right)=\omega\left(e_{2} \otimes e_{1}\right)=\frac{1}{2}, \quad \omega\left(e_{2} \otimes e_{2}\right)=0, \\
& \omega\left(e_{1} \otimes e_{1}\right)=0, \quad \omega\left(e_{1} \otimes e_{2}\right)=\omega\left(e_{2} \otimes e_{1}\right)=0, \quad \omega\left(e_{2} \otimes e_{2}\right)=1 .
\end{aligned}
$$

Лемма 4.1. Пусть выполнено одно из условий (i), (ii), (iii). Тогда транслячионно-инвариантное основное состояние для марковского гамильтониана диагонализуемо.

ДокАЗАтЕльСтво. Рассмотрим случай (i). Тогда основное состояние $\omega$ удовлетворяет условию (14). Легко видеть, что для любого $n \in \mathbb{N}$

$$
\begin{aligned}
\omega(\underbrace{e_{1} \otimes \cdots \otimes e_{1}}_{n}) & =1, \\
\omega\left(e_{i_{1}} \otimes \cdots \otimes e_{i_{n}}\right. & =0, \quad \text { если хотя бы один из } i_{k} \text { равен } 2 .
\end{aligned}
$$

Из условия (14) и положительности $\omega$ следует

$$
\omega\left(e_{3}\right)=\omega\left(e_{4}\right)=0 \text {. }
$$

Покажем, что $\omega\left(e_{i} \otimes e_{j}\right)=0$, если хотя бы один из $i$ или $j$ равен 3 или 4 . Определим положительные функционалы $\varphi(\cdot)$ и $\psi(\cdot)$, полагая

$$
\varphi(x)=\omega\left(e_{1} \otimes x\right), \quad \psi(x)=\omega\left(e_{2} \otimes x\right), \quad x \in M_{2}(\mathbb{C}) .
$$

Из (15) и положительности этих функционалов следует

$$
\varphi\left(e_{3}\right)=\varphi\left(e_{4}\right)=0, \quad \psi(x)=0 \quad \forall x \in M_{2}(\mathbb{C}) .
$$

Учитьвая (16), (17) и положительность $\omega$, имеем

$$
\omega\left(e_{3} \otimes e_{4}\right)=\omega\left(e_{4} \otimes e_{4}\right)=0 .
$$


Из (16)-(18) вытекает требуемое равенство.

Предположим, что для состояния, удовлетворяющего условию (14) для некоторого $n \in \mathbb{N}$, справедливо равенство

$$
\omega\left(e_{i_{1}} \otimes \cdots \otimes e_{i_{n}}\right)=0, \quad \text { если хотя бы один из } i_{k} \text { равен } 3 \text { или } 4,
$$

где $k \in\{1, \ldots, n\}$. Покажем, что тогда это равенство верно и для $n+1$. Для любого $k \in\{1, \ldots, n+1\}$ определим положительные функционалы следующим образом:

$$
\begin{aligned}
& \varphi_{k}\left(x_{1} \otimes \cdots \otimes x_{n}\right)=\omega\left(x_{1} \otimes \cdots \otimes x_{k-1} \otimes e_{1} \otimes x_{k} \otimes \cdots \otimes x_{n}\right), \\
& \psi_{k}\left(x_{1} \otimes \cdots \otimes x_{n}\right)=\omega\left(x_{1} \otimes \cdots \otimes x_{k-1} \otimes e_{2} \otimes x_{k} \otimes \cdots \otimes x_{n}\right),
\end{aligned}
$$

где $x_{i} \in M_{2}(\mathbb{C}), i=1, \ldots, n$.

Легко видеть, что $\varphi_{k}$ удовлетворяет условию (14). Следовательно, по предположению имеем

$$
\varphi_{k}\left(e_{i_{1}} \otimes \cdots \otimes e_{i_{n}}\right)=0
$$

если хотя бы один $i_{l}, l \in\{1, \ldots, n+1\}$, равен 3 или 4. Из положительности $\psi_{k}$ и условия (15) вытекает

$$
\psi_{k}\left(x_{1} \otimes \cdots \otimes x_{n}\right)=0 \quad \forall x_{i} \in M_{2}(\mathbb{C}), \quad i=1, \ldots, n
$$

Итак, учитьвая (15), (19)-(21) и положительность $\omega$, получим требуемое равенство. Таким образом, по индукции заключаем, что состояние, удовлетворяющее условию (14), диагонализуемо.

Аналогичным образом рассматриваются остальные случаи. Лемма доказана.

Из этой леммы немедленно следует следующая

ТЕОРемА 4.1. Пусть выполнено одно из условий (i), (ii), (iii). Тогда для марковского гамильтониана Н существует единственное трансляционно-инвариантное основное состояние.

Благодарность. Авторы выражают благодарность рецензенту за полезные замечания, которые, несомненно, способствовали улучшению текста статьи. Также отметим, что пример в замечании 3 указан рецензентом. 


\section{СПИСОК ЦИТИРОВАННОЙ ЛИТЕРАТУРЫ}

[1] Powers R. Representation of uniformly hyperfinite algebras and their assosiated von Neumann rings // Ann. of Math. 1967. V. 81. P. 138-171.

[2] Блехер П. М. Некоторые применения факторов // Дж. фон Нейман "Избранные труды по функциональному анализу II". М.: Наука, 1987. С. 353-359.

[3] фон Нейман Дж. Избранные труды по функциональному анализу II. М.: Наука, 1987.

[4] Браттели У., Робинсон Д. Операторные алгебры и квантовая статистическая механика. М.: Мир, 1982.

[5] Connes A. Une classification des facteurs de type III // Ann. Ecole Norm. Sup. 1973. V. 6. P. 133-252.

[6] Pillis J. Non commutative Markov processes // Trans. Amer. Math. Soc. 1966. V. 125. № 2. P. 264-279.

[7] Аккарди Л. О некоммутативном марковском свойстве // Функцион. анализ и его прилож. 1975. Т. 8. №1. С. 1-8.

[8] Шухов А. Г. Об энтропии диагонализуемых состояний неймановских алгебр // Функцион. анализ и его прилож. 1980. Т. 14. №2. С. 95-96.

[9] Синай Я. Г. Теория фазовых переходов. Строгие результаты. М.: Наука, 1980.

[10] Bratteli O., Robinson D. Operator algebras and Quantum Statistical Mechanics II. Berlin: Springer Verlag, 1981.

[11] Araki H. On uniqueness of KMS states of one-dimensional quantum lattice systems // Comm. Math. Phys. 1975. V. 44. P. 1-7.

[12] Kriger W. On the finitary isomorphism of Markov shifts that have finite coding time // Z. Wahr. verw. Geb. 1983. V. 65. P. 323-328.

[13] Golodets V. Ya., Neshveyev S. V. Non-Bernoullian K-systems // Comm. Math. Phys. 1998. V. 195. P. 213-232.

[14] Stratila S. Modular Theory in Operator Algebras. Bucuresti: Abacus Press, 1981.

[15] Glimm J. Type I $C^{*}$-algebras // Ann. of Math. 1961. V. 73. P. 572-612.

[16] Murakami T., Yamagami S. On types of quasifree representations of Clifford algebras // Publ. RIMS. 1995. V. 31. P. 33-44.

Национальный университет Узбекистана, г. Ташкент

Поступило

E-mail: root@im.tashkent.su, far 75 m@yandex.ru 